\title{
Identification of FDA Approved Drugs Targeting COVID-19 Virus by Struc- ture-Based Drug Repositioning
}

Ayman B. Farag ${ }^{1 *}$, Ping Wang ${ }^{1 *}$, Mahmoud S. Ahmed ${ }^{1}$ and Hesham A. Sadek ${ }^{1,}$ $2,3,4,5$

Departments of Internal Medicine ${ }^{1}$, Molecular Biology $^{2}$, and Biophysics ${ }^{3}$, and Center for Regenerative Science and Medicine ${ }^{5}$, University of Texas Southwestern Medical Center, Dallas, Texas 75390, USA

${ }^{*}$ Authors contributed equally. 


\title{
Corresponding authors:
}

\author{
Mahmoud S. Ahmed, PhD \\ Department of Internal Medicine/Division of Cardiology \\ University of Texas Southwestern Medical Center \\ 6000 Harry Hines Blvd \\ Dallas, Texas, 75390, USA
}

Fax number: 214-648-1450

Telephone number: 214-648-1413

Email: Mahmoud.ahmed@utsouthwestern.edu

Hesham A. Sadek, MD, PhD

Departments of Internal Medicine/Division of Cardiology, Molecular Biology and Biophysics

University of Texas Southwestern Medical Center

6000 Harry Hines Boulevard

Dallas, Texas, 75390, USA

Fax number: 214-648-1450

Telephone number: 214-648-1413

Email: hesham.sadek@utsouthwestern.edu 


\section{Abstract:}

The new strain of Coronaviruses (SARS-CoV-2), and the resulting Covid-19 disease has spread swiftly across the globe after its initial detection in late December 2019 in Wuhan, China, resulting in a pandemic status declaration by WHO within 3 months. Given the heavy toll of this pandemic, researchers are actively testing various strategies including new and repurposed drugs as well as vaccines. In the current brief report, we adopted a repositioning approach using insilico molecular modeling screening using FDA approved drugs with established safety profiles for potential inhibitory effects on Covid-19 virus. We started with structure based drug design by screening more than 2000 FDA approved drugs against Covid-19 virus main protease enzyme $\left(\mathrm{M}^{\text {pro }}\right)$ substrate-binding pocket to identify potential hits based on their binding energies, binding modes, interacting amino acids, and therapeutic indications. In addition, we elucidate preliminary pharmacophore features for candidates bound to Covid-19 virus $\mathrm{M}^{\text {pro }}$ substratebinding pocket. The top hits include anti-viral drugs such as Darunavir, Nelfinavir and Saquinavir, some of which are already being tested in Covid-19 patients. Interestingly, one of the most promising hits in our screen is the hypercholesterolemia drug Rosuvastatin. These results certainly do not confirm or indicate antiviral activity, but can rather be used as a starting point for further in vitro and in vivo testing, either individually or in combination. 


\section{Introduction:}

The new strain for Coronaviruses (CoVs) was identified in late December 2019 named SARS-CoV-2 (Covid-19 virus) resulted in a massive outbreak initially in Wuhan, China and propagated to different nations around the globe. On March 11, World Health Organization (WHO) declared the resulting disease named COVID-19 as a pandemic [1]. Coronavirus cases are estimated at 169,112 with 6,494 death cases as of March 15th 2020 [2]. Clinical efforts to discover potential vaccines and therapeutics are still ongoing with no clear treatment or prophylaxis for COVID-19 in sight. It is safe to say that a sufficient understanding of SARSCoV-2, and the full clinical picture of the resulting COVID-19 disease will take some time. Similarly, developing and widely distributing effective vaccines or novel antiviral drugs is unlikely to occur during this season, which leaves healthcare systems vulnerable, and risks high mortality rates. Alternatively, drugrepurposing strategies can create viable path towards identification of potential therapeutics with established safety profiles that can be used individually or in combinations for targeting molecular regulators of replication or the survival of SARS-CoV-2. While these strategies are unlikely to provide immunity or cure, they may identify therapeutics that can alter the clinical course of COVID-19, especially in critically ill patients[3-8].

The betacoronavirus genome encodes structural proteins, including the glycosylated spike (S) protein that serves as a major inducer of host immune responses. 
The spike protein mediates host cell invasion via binding to angiotensinconverting enzyme 2 (ACE2) (a homolog of angiotensin converting enzyme ACE) which is a membrane bound carboxypeptidase). The cellular invasion process appears to be mediated by priming of $S$ protein facilitated by the host cellproduced serine protease TMPRSS2 . In addition, the viral genome also encodes nonstructural proteins including RNA-dependent RNA polymerase (RdRp), coronavirus main protease $\left(\mathrm{M}^{\text {pro }}\right)$, and papain-like protease (PLpro) $[5,9,10]$. Therefore, targeting ACE2, TMPRSS211, RdRp, $\mathrm{M}^{\text {pro }}$, and PLpro, as individual targets, or in combination, is a viable strategy for repurposed drugs. To that end, different drug repurposing efforts have been executed starting with data driven framework coupled with in vitro assays to show the potential of a poly-ADPribose polymerase 1 (PARP1) inhibitor, CVL218, currently in a Phase I clinical trial, may serve as a potential drug candidate to inhibit SARS-CoV-2 replication in a dose-dependent manner and with no obvious cytopathic effect. CVL218 showed potential binding affinity of the $\mathrm{N}$-terminal domain of nucleocapsid $(\mathrm{N})$ protein of COVID-19 virus via in-silico analysis [11].

Herein, our structure based drug design approach is focused on targeting COVID-19 virus $\mathrm{M}^{\text {pro }}$ based on the elegant work that resulted in solving the crystal structure of COVID-19 $\mathrm{M}^{\text {pro }}$ in complex with an inhibitory peptide N3 (PDB ID: 6LU7). In particular, that inhibitory peptide binds the substrate-binding pocket of COVID-19 $\mathrm{M}^{\text {pro }}$. This domain was the focus of our screen with regards to the potential hydrophobic binding domain and considering the hydrogen bond network. 
Starting with the published crystal structure provided us with structural insights for of the catalytic binding domain and active draggable sites, elucidating free binding energies with respect to binding affinity and interactions, as shown in figure 1. The purpose of the current study is to accelerate the use of clinically viable approved therapeutics in the setting of a pandemic, and highlights some important pharmacophore features for generation of novel molecules that target SARS-CoV-2.

\section{Results and Discussion:}

The structural analysis for the peptide ligand co-crystallized along with COVID-19 virus $\mathrm{M}^{\text {pro }}$ substrate-binding pocket revealed the significance of hydrophobic interactions and hydrogen bond network comprising His163, His164, Glu166, GIn189, and Thr190[8]. In-silico molecular modeling for >2000 FDA approved drugs showed the top 11 hits based on their S score, binding affinity, interacting amino acids, and binding mode to fit the main protease pocket with respect to the reported clinical indication, as shown in Table 1. The rest of the top 100 hits are listed in Table S1.

Our two and three-dimensional analysis for the top hits is shown in figures 2 and 3. Darunavir, a known antiviral with protease inhibitory mode of action [12], showed the best binding affinity in terms of hydrophobic-hydrophobic and hydrogen bond interactions with His163, Glu166, and Thr190 at S score -14.03040 
$\mathrm{Kcal} / \mathrm{mol}$. The top candidates also included antivirals such as Nelfinavir and Saquinavir at S score ranging from $-13.4200 \mathrm{Kcal} / \mathrm{mo}$ to $-12.0917 \mathrm{Kcal} / \mathrm{mol}$.

The current repurposing study also includes other drugs approved for different clinical indications. For example, Moexipril, an ACE inhibitor [13], showed a proper binding mode via hydrophobic interactions and a hydrogen bond through a carboxylic acid moiety with Gln189 at S score $-13.2142 \mathrm{Kcal} / \mathrm{mol}$. Daunorubicin and Mitoxantrone are representatives for anthracene glycosidic chemotherapeutic agents[14, 15]. Daunorubicin showed potential binding affinity via hydrophobic interactions and hydrogen bonding with Asn142 and Glu166. Mitoxantrone showed hydrophobic interactions and a network of hydrogen bonds with His41, His164, Asp187, Thr190, and GIn192. The anthracene derivatives findings suggests that glycoside based derivatives can target the substrate-binding pocket of COVID-19 virus $\mathrm{M}^{\text {pro }}$. However administration of chemotherapeutic agents can lead to significant adverse effects and is unlikely to be of any clinical utility in critically ill COVID-19 patients. Metimazole, a pyrazolone based derivative [16], showed a potential binding affinity via hydrogen bonds between a sulphonic acid moiety and His 163 and Ser144 in addition to pi-pi interaction with His41. Although theoretically metamizole might have clinical utility here, it is also not an ideal candidate due to its association with agranulocytosis, which would be an unwelcomed side effect in COVID-19 patients. Bepotastine, an anti-histamine $[17,18]$, exhibited proper binding affinity in the main substrate-binding pocket via hydrophobic interactions and hydrogen bonding through a carboxylic acid moiety 
with Gln189. Atovaquone, an anti-malarial drug [19] showed a potential binding affinity with no hydrogen bonds with an S score $-8.4159 \mathrm{Kcal} / \mathrm{mol}$. This might complement the current use of chloroquine, another anti-malarial drug, which shares the same scaffold features of atovaquone, to elevate endosomal $\mathrm{pH}$ and interfere with ACE2 glycosylation. One of the promising drugs from the current screen that might warrant further investigation is Rosuvastatin, which belongs to the statins class of antihyperlipidemic drugs [20]. We found that Rosuvastatin displayed an excellent binding affinity in terms of free energy with an S score of $12.3096 \mathrm{Kcal} / \mathrm{mol}$. The Rosuvastatin skeleton filled the entire substrate-binding pocket via hydrophobic interactions and hydrogen bonding with Gly143 and Glu166.

In the current report, we show that structure based drugs design for the top hits elucidated the following preliminary pharmacophore features: (1) The entire pocket needs hydrophobic features or extended phenyl moieties to maintain a proper binding affinity such as in case of anthracene derivatives. (2) A network of hydrogen bonds (whether donors or acceptors) is a significant factor especially with amino acids Glu166, Gln189, His163, and His164. (3) The presence of terminal sulphonic acid and/or carboxylic acid moieties (Bepotastine, Moexipril, Metimazole, and Rosuvastatin) can act as bio-isosteric moieties to the phosphate groups that can be found in antiviral drugs. One of the interesting studies that can be carried out in the future is to identify FDA drugs that can target Cys 145 
within the substrate-binding pocket covalently, especially given that the cocrystallized inhibitor is peptide in nature with covalent mode of binding.

Therefore, screening the FDA approved drug library against the COVID-19 virus $\mathrm{M}^{\text {pro }}$ substrate-binding pocket can provide valuable insights to fast-track clinical trials for drugs with an established safety profile. Several top hits from our screen, including Rosuvastatin, can potentially be safely used in COVID-19 patient, however additional studies are needed to determine the efficacy of these hits.

\section{Materials and Methods:}

FDA approved small molecules preparation: The U.S. FDA approved drug database was downloaded (drugbank.ca) and 3D structures were energy minimized using MMFF94 force field.

X-ray crystal structure preparation: Crystal structure of COVID-19 virus $\mathrm{M}^{\text {pro }}$ co-crystallized with an inhibitor has been resolved and accessed via PDB ID: 6LU7. A box was assigned for docking procedures within the hydrophobic binding domain of the peptide inhibitor.

Structure based in-silico screening and scoring: The whole energy minimized library was enrolled in docking simulations using Molecular Operating Environ- 
ment (MOE) along with PDB ID: 6LU7. The energy-minimized drugs underwent protonation states to add the missing hydrogens for proper ionization states. MOE docking module used to evaluate the favorable binding conformers based on London dG scoring method to estimate energy profile based on the binding affinity with respect to hydrophobic-hydrophobic interactions, hydrogen bonding, pi-pi interactions, and ionic interactions [21].

Each drug gave 10 possible docked poses. The ideal pose for each drug was selected according to the similarity of its binding mode in the binding pocket to that of the co-crystallized peptide. Two dimensional diagrams were generated using MOE tools, while three dimensional figures were generated using pymol.

\section{Conclusion:}

In the current report, a structure based drug repositioning strategy was adopted to repurpose FDA approved drugs targeting COVD-19 $\mathrm{M}^{\text {pro }}$ substrate-binding pocket which revealed a number of potentially usable drugs that may have clinical utility in COVID-19. Although these are promising findings, these results need further biological in vitro and in vivo validation. 


\section{Figures:}

A

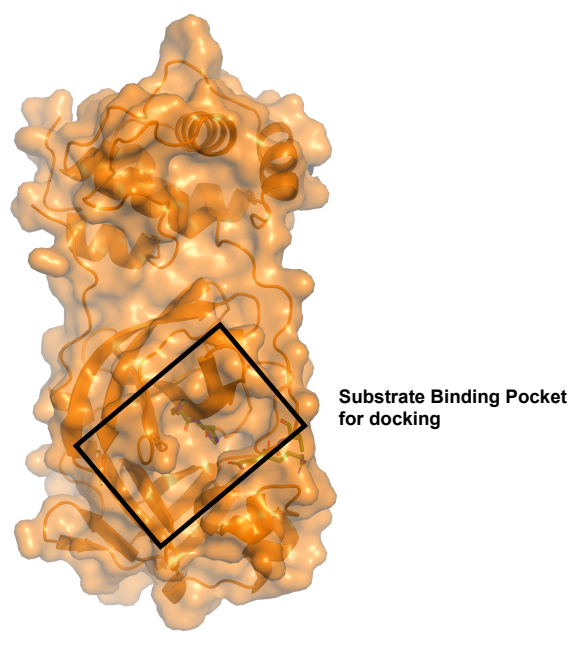

PDB ID: 6lu7
B
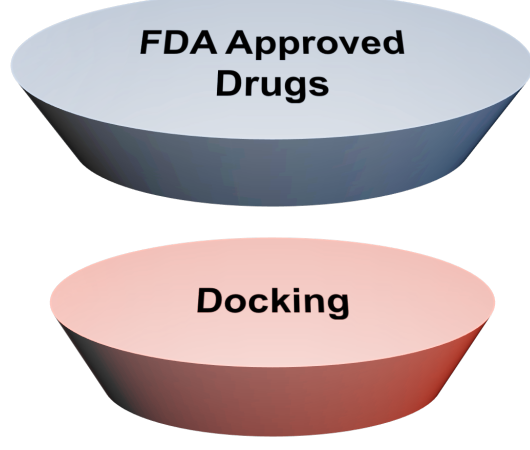

Top Candidates

Figure 1. Workflow: A) Crystal structure of COVID-19 virus $\mathrm{M}^{\text {pro }}$ highlighting the substrate binding-pocket which was used for docking. B) Schematic diagram for in-silico drug repositioning. 


\begin{tabular}{|l|l|l|}
\hline Drug & S score (Kcal / mol) & Clinical Indication \\
\hline Darunavir & -14.0304 & Antiviral \\
\hline Mitoxantrone & -13.8100 & Anticancer \\
\hline Nelfinavir & -13.4200 & Antiviral \\
\hline Moexpril & -13.2442 & Anti-hypertensive \\
\hline Daunorubicin & -12.5009 & Anticancer \\
\hline Rosuvastatin & -12.3096 & Anti-hypercholesterolemia \\
\hline Saquinavir & -12.0917 & Antiviral \\
\hline Metamizole & -11.6652 & Anti-inflammatory \\
\hline Bepotastine & -10.6350 & Anti-histaminic \\
\hline Benzonatate & -10.4759 & Anti-tussive \\
\hline Atovaqoune & -8.1459 & \\
\hline
\end{tabular}

\section{Table 1. List of top drugs docked to COVID-19 virus $M^{\text {pro }}$ substrate-binding} pocket (1-12) based on S score. 

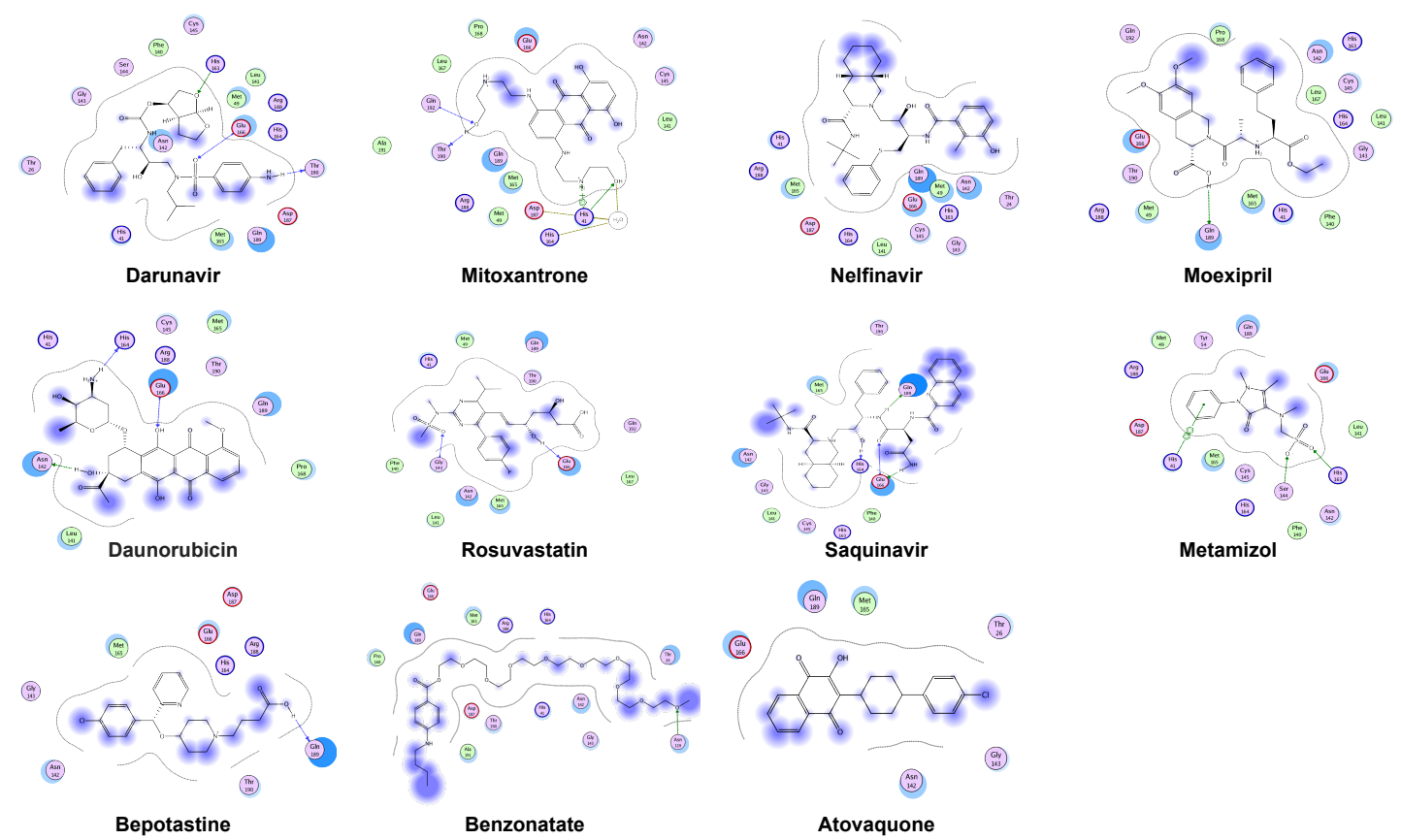

(i.i) (:i)

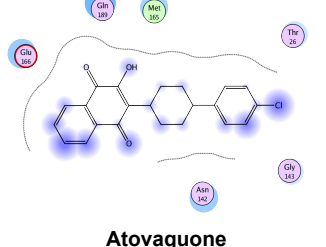

Figure 2. Two-dimensional presentation of docking poses for top 11 candidates. Blue arrows are backbone hydrogen bonds and green arrows are the side chain hydrogen bonds. 


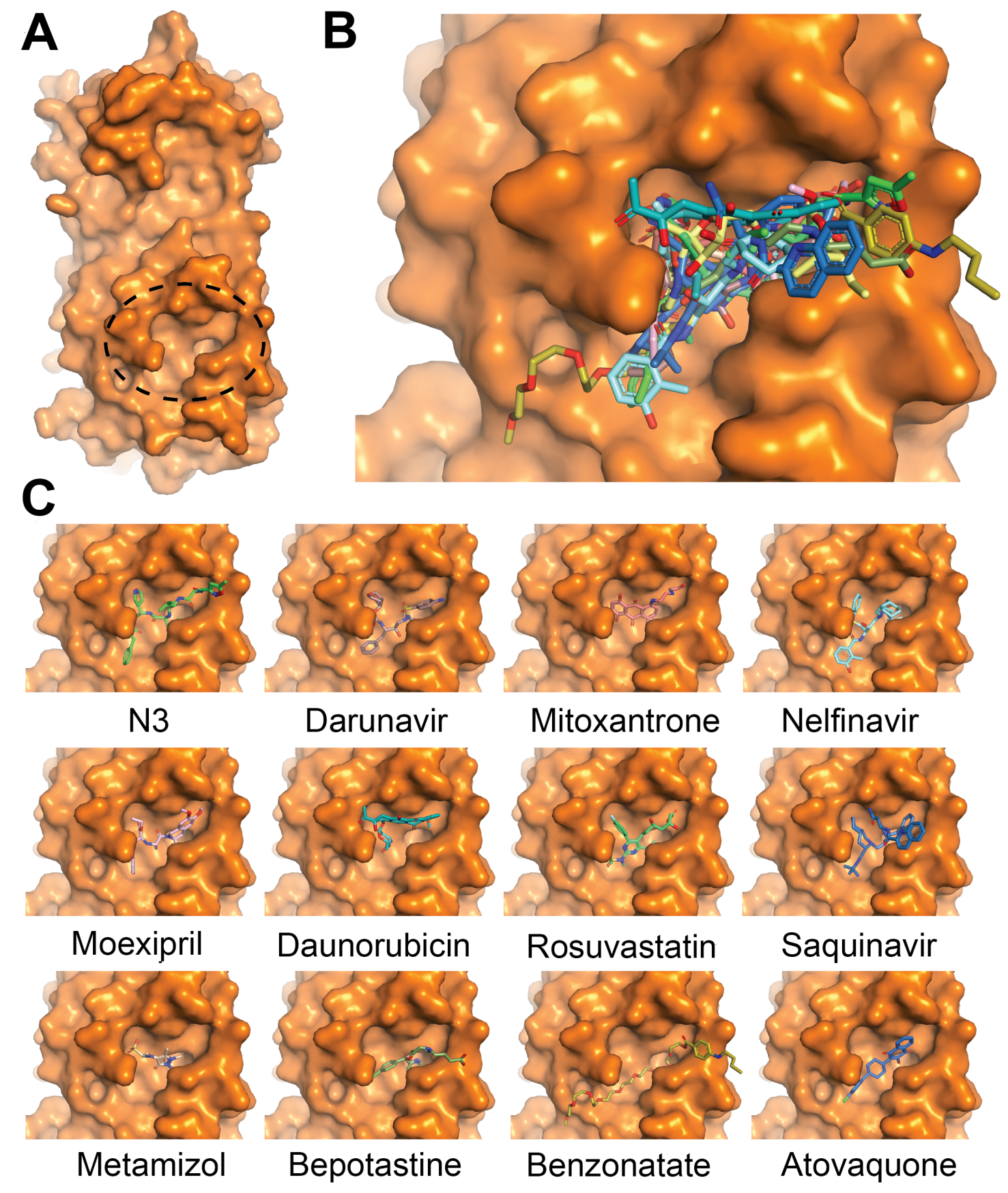

Figure 3. Docked positions to the substrate binding-pocket of COVID-19 virus $M^{\text {pro: }}$ A) 3D presentation of the pocket. B) Overlayed docked positions of all 11 
top hits in addition the previously co-cryslatlized inhibitory peptide N3. C) Individual docking positions.

\section{Supporting Information:}

Table S1. List of the remaining top 100 drugs based on S score.

\begin{tabular}{|l|l|}
\hline Drug & S score (Kcal / mol) \\
\hline Dipiverfin & -8.1378 \\
\hline Defroxamine & -8.1225 \\
\hline Dobutamine & -8.1108 \\
\hline Metipanolol & -8.0286 \\
\hline Ziprosidone & -8.0030 \\
\hline Cladribine & -7.9624 \\
\hline Almitrine & -7.9462 \\
\hline Epoprostenol & -7.8723 \\
\hline Famciclovir & -7.8672 \\
\hline Fluvastatine & -7.8443 \\
\hline Ticonazole & -7.8416 \\
\hline Delviradine & -7.8185 \\
\hline
\end{tabular}




\begin{tabular}{|l|l|}
\hline Drug & S score (Kcal / mol) \\
\hline Methantheline & -7.7925 \\
\hline Mefloquine & -7.7520 \\
\hline Diatrizoate & -7.6401 \\
\hline Iloperidone & -7.6082 \\
\hline Sapropterin & -7.5764 \\
\hline Vincristine & -7.5485 \\
\hline Oxamniquine & -7.54058 \\
\hline Teniposide & -7.5293 \\
\hline Abacavir & -7.4897 \\
\hline Zoledronic acid & -7.4834 \\
\hline Etoposide & -7.3944 \\
\hline Carbetocin & -7.4235 \\
\hline vesoriconazole & -7.3694 \\
\hline Timolol & \\
\hline Loratadine & \\
\hline varimetasone & -7.2958 \\
\hline
\end{tabular}




\begin{tabular}{|l|l|}
\hline Drug & S score (Kcal / mol) \\
\hline Tipranvir & -7.2732 \\
\hline Chloroquine & -7.2639 \\
\hline Indometacin & -7.2021 \\
\hline Meloxicam & -7.2002 \\
\hline Labetolol & -7.2001 \\
\hline Deslanoside & -7.1533 \\
\hline Leflunomide & -7.1231 \\
\hline Pimozide & -7.0937 \\
\hline Repaglinide & -7.0160 \\
\hline Furosemide & -6.2281 \\
\hline Candoxatril & -6.9690 \\
\hline Indapamide & -6.8322 \\
\hline Cimvastatin & -6.8165 \\
\hline Donedarone & -6.6003 \\
\hline & \\
\hline & \\
\hline & \\
\hline
\end{tabular}




\begin{tabular}{|l|l|}
\hline Drug & S score (Kcal / mol) \\
\hline Indinavir & -6.2012 \\
\hline Paricalcitol & -6.1779 \\
\hline Imodium & -6.1374 \\
\hline Bexarotene & -6.0809 \\
\hline Clofarabine & -6.0798 \\
\hline Betamethasone & -5.9249 \\
\hline Bricodar & -5.7965 \\
\hline Nafareline & -5.7426 \\
\hline Mebendazol & -5.7426 \\
\hline Levocabastine & -5.6281 \\
\hline Losartan & -5.3706 \\
\hline Omapatrilate & -5.3388 \\
\hline Flecainide & -4.1052 \\
\hline
\end{tabular}




\section{References:}

1. Malik, Y.S., et al., Emerging novel coronavirus (2019-nCoV)-current scenario, evolutionary perspective based on genome analysis and recent developments. Vet Q, 2020. 40(1): p. 68-76.

2. Lee, P.I. and P.R. Hsueh, Emerging threats from zoonotic coronavirusesfrom SARS and MERS to 2019-nCoV. J Microbiol Immunol Infect, 2020.

3. Du, L., et al., The spike protein of SARS-CoV--a target for vaccine and therapeutic development. Nat Rev Microbiol, 2009. 7(3): p. 226-36.

4. Wrapp, D., et al., Cryo-EM structure of the 2019-nCoV spike in the prefusion conformation. Science, 2020. 367(6483): p. 1260-1263.

5. Hoffmann, M., et al., SARS-CoV-2 Cell Entry Depends on ACE2 and TMPRSS2 and Is Blocked by a Clinically Proven Protease Inhibitor. Cell, 2020.

6. Anthony, S.J., et al., Global patterns in coronavirus diversity. Virus Evol, 2017. 3(1): p. vex012.

7. Anand, K., et al., Coronavirus main proteinase (3CLpro) structure: basis for design of anti-SARS drugs. Science, 2003. 300(5626): p. 1763-7.

8. Wang, F., et al., Structure of Main Protease from Human Coronavirus NL63: Insights for Wide Spectrum Anti-Coronavirus Drug Design. Sci Rep, 2016. 6: p. 22677.

9. Gorbalenya, A.E., et al., Nidovirales: evolving the largest RNA virus genome. Virus Res, 2006. 117(1): p. 17-37.

10. Baez-Santos, Y.M., S.E. St John, and A.D. Mesecar, The SARScoronavirus papain-like protease: structure, function and inhibition by designed antiviral compounds. Antiviral Res, 2015. 115: p. 21-38.

11. Chang, K.O., et al., Antiviral Drug Discovery: Norovirus Proteases and Development of Inhibitors. Viruses, 2019. 11(2).

12. $\mathrm{Li}, \mathrm{D}$., et al., Investigation on the mechanism for the binding and drug resistance of wild type and mutations of G86 residue in HIV-1 protease complexed with Darunavir by molecular dynamic simulation and free energy calculation. J Mol Model, 2014. 20(2): p. 2122.

13. Kalasz, H., et al., Metabolism of moexipril to moexiprilat: determination of in vitro metabolism using HPLC-ES-MS. Med Chem, 2007. 3(1): p. 101-6.

14. Pang, B., et al., Drug-induced histone eviction from open chromatin contributes to the chemotherapeutic effects of doxorubicin. Nat Commun, 2013. 4: p. 1908.

15. Wu, C.C., et al., On the structural basis and design guidelines for type II topoisomerase-targeting anticancer drugs. Nucleic Acids Res, 2013. 41(22): p. 10630-40.

16. Garcia-Martin, E., et al., Genetic determinants of metamizole metabolism modify the risk of developing anaphylaxis. Pharmacogenet Genomics, 2015. 25(9): p. 462-4. 
17. Takahashi, D., et al., Backbone and side-chain (1)H, (1)(5)N, and (1)(3)C resonance assignments of Norwalk virus protease. Biomol NMR Assign, 2012. 6(1): p. 19-21.

18. Takahashi, H., A. Ishida-Yamamoto, and H. lizuka, Effects of bepotastine, cetirizine, fexofenadine, and olopatadine on histamine-induced wheal-and flare-response, sedation, and psychomotor performance. Clin Exp Dermatol, 2004. 29(5): p. 526-32.

19. Hughes, W., et al., Comparison of atovaquone (566C80) with trimethoprim-sulfamethoxazole to treat Pneumocystis carinii pneumonia in patients with AIDS. N Engl J Med, 1993. 328(21): p. 1521-7.

20. Jones, P.H., et al., Comparison of the efficacy and safety of rosuvastatin versus atorvastatin, simvastatin, and pravastatin across doses (STELLAR* Trial). Am J Cardiol, 2003. 92(2): p. 152-60.

21. Naim, M., et al., Solvated interaction energy (SIE) for scoring proteinligand binding affinities. 1. Exploring the parameter space. J Chem Inf Model, 2007. 47(1): p. 122-33. 